\title{
Kinect-Based Human Gait Recognition Using Locally Linear Embedded and Support Vector Machine
}

\author{
(Pengenalpastian Gait Manusia Berasaskan Gerakan Menggunakan Terbenam Linear Setempat dan Mesin \\ Penyokong Vektor) \\ Rohilah Sahak, Nooritawati Md Tahir*, Ahmad Ihsan Mohd Yassin \& Fadhlan Hafizhelmi Kamaruzaman \\ Faculty of Electrical Engineering, Universiti Teknologi MARA (UiTM), Shah Alam, Selangor, Malaysia
}

\begin{abstract}
Recognition of human gait could be performed effectively provided that significant gait features are well extracted along with effective recognition process. Thus, the gait features should be selected or optimized appropriately for optimal accuracy during recognition. Therefore, in this research, optimization of gait features for both oblique and frontal view are evaluated for recognition purpose using Locally Linear Embedded (LLE) along with multi-class Support Vector Machine (SVM). Firstly, dynamic gait features for one gait cycle are extracted from each subject's walking gait that is acquired using Kinect sensor. Next, the extracted gait features were then optimized using LLE known as DG-LLE and further classified by multi-class SVM with Error Correcting Output Code (ECOC) algorithm. Further, to validate the effectiveness of LLE as optimization technique, the proposed method is then compared with another two gait features namely the original gait features known as DG and optimization using Principal Component Analysis labeled as DG-PCA. Results showed that the optimization based on DG-LLE outperformed the other two methods namely DG and DG-PCA for both oblique and frontal views. In addition, DG-LLE method contributed as the highest recognition rate for both frontal and oblique views. Results also confirmed that the accuracy rate for frontal view is higher specifically $98.33 \%$ as compared to oblique view with $94.67 \%$.
\end{abstract}

Keywords: Frontal gait recognition; kinect; locally linear embedded; support vector machine

ABSTRAK

Pengecaman manusia melalui gaya berjalan boleh dijalankan secara lebih efektif dengan menggunakan fitur yang penting serta proses pengecaman yang efektif. Oleh yang demikian, fitur gaya berjalan yang optimum perlu dipilih untuk mendapatkan ketepatan yang terbaik semasa proses pengecaman. Maka dalam kajian ini, pengoptimuman fitur gaya berjalan untuk pandangan oblik dan pandangan hadapan dinilai untuk tujuan pengecaman, menggunakan kaedah Terbenam Linear Setempat (LLE) dan Mesin Penyokong Vektor (SVM). Pertama, fitur dinamik satu kitaran gaya jalan subjek diekstrak menggunakan sensor Kinect. Seterusnya, fitur yang diekstrak dioptimumkan menggunakan kaedah LLE yang dikenali sebagai DG-LLE diikuti dengan fasa pengelasan menggunakan algoritma Mesin Penyokong Vektor pelbagai kelas dan Kod Keluaran Pembetul Ralat (ECOC). Selanjutnya, untuk mengesahkan keberkesanan LLE sebagai kaedah pengoptimuman, kaedah tersebut dibandingkan dengan dua jenis fitur gaya berjalan yang dikenali sebagai fitur asal gaya berjalan iaitu DG dan fitur yang dioptimumkan menggunakan kaedah Komponen Analysis Utama yang dilabel sebagai DG-LLE. Keputusan kadar pengecaman menunjukkan bahawa pengoptimuman menggunakan DG-LLE adalah lebih tinggi berbanding kaedah DG dan DG-PCA, bagi kedua-dua pandangan oblik dan pandangan hadapan. Selain itu, bagi gaya berjalan pandangan hadapan, DG-LLE memberikan kadar pengecaman yang lebih tinggi berbanding gaya berjalan pandangan sisi iaitu dengan kadar ketepatan bernilai 98.33\% bagi pandangan hadapan berbanding $94.67 \%$ bagi pandangan oblik.

Kata kunci: Pengenalpastian gaya berjalan pandangan hadapan; gerakan; terbenam linear setempat, mesin penyokong vektor

\section{INTRODUCTION}

The study of gait as features in human recognition has been extensively carried out due to its advanced characteristics in biometrics namely fingerprints, iris (Mei et al. 2007), and voice recognition (Sharrif \& Theong 1991). Each individual gait has its unique characteristics of its own that are difficult to emulate and to recognise human from their gait does not require physical contact between human and the recognition device as well (Yoo \& Nixon 2011).

Gait analysis can be divided into model-based analysis and model-free analysis. In the model-free analysis, information from the silhouette shape is extracted for further analysis. On the other hand, the model-based analysis requires data from body structure. As opposed to model-based analysis, the model-free analysis involves low computational 
cost and gait feature representation. However, the modelfree analysis is limited to circumstances such as occlusion and clothing conditions (Hosseini \& Nordin 2013; Han \& Bhanu 2004; Rida, Jiang \& Marcialis 2016). In order to overcome these limitations, numerous researches have been conducted, for instance, researchers focused on generating potential extracted features that in return reduced the effect of occlusion and clothing conditions (Han \& Bhanu 2006; Sengupta et al. 2013; Kochhar et al. 2013; Shirke, Pawar \& Shah 2014). Moreover, the optimisation of gait features with various feature optimisation methods are being developed due to the large dimension of extracted gait features. These feature optimisation methods are also meant to improve recognition performances (Hofmann \& Rigoll 2013; Lam et al. 2011; Hofmann et al. 2014b; Sivapalan et al. 2012; Ekinci 2006; Ekinci 2007; Fazli et al. 2011; Sivapalan et al. 2011; Hofmann et al. 2014a). The common methods used are Principal Component Analysis (PCA) and Linear Discriminant Analysis (LDA). These methods were proven to be effective along with classifiers namely Support Vector Machine (SVM) and k-Nearest Neighbour (KNN) (Hofmann et al. 2014a; Fazli, et al. 2011; Hofmann \& Rigoll 2013).

On the contrary, model-based analysis is robust to these limitations. These limitations do not affect the recognition of the model-based analysis. Unfortunately, the model-based analysis requires high computational cost for modelling and tracking the human gait. Numerous studies on generating model fitting with low computational cost have been carried out, however, as Kinect was introduced by Preis et al. (Preis et al. 2012) in 2012, the motivation behind these studies have migrated to extracting potential gait features and pattern classifier (Preis et al. 2012; Naresh Kumar and Venkatesh Babu 2012; Araujo et al. 2013; Gianaria et al. 2013; Sadhu and Konar 2014; Andersson and Araújo 2014; Jiang et al. 2014; Gianaria et al. 2014). Kinect is a low-cost motion sensing device capable of modelling and tracking human motion, for instance, posing, walking, running or other similar actions and automatically provides information of the skeleton joints of the walking human in both $3 \mathrm{D}$ and $2 \mathrm{D}$ space. As compared to a standard video camera, Kinect is able to generate skeleton joints, accurately, without involving complex processes. Studies done by Preis et al. 2012; Kumar \& Babu 2012; Araujo and Andersson 2013; Gianaria et al. 2014; Chang \& Nam 2013 have proven that features extracted using Kinect are indeed reliable for human recognition as these studies reported good recognition performance. Therefore, in this study, video sequences of human walking gait captured using Kinect was employed in the data acquisition stage.

Investigation on extracting potential features is still ongoing which can further widen the potential for feature optimisations. Hence, in this study, we deem further to develop feature extraction method of dynamic human gait in both oblique and frontal view followed by optimisation using Locally Linear Embedded (LLE) based on 30 subjects. Consequently, the multi-class SVM with Error Correcting Output Code (ECOC) algorithm is employed as pattern recognition. In order to verify the performance of LLE, both original gait features along with PCA as the feature extraction is conducted. Recognition of these three approaches are computed and compared to acquire the best feature optimisation method.

RELATED WORKS

\section{HUMAN GAIT RECOGNITION WITH KINECT}

Preis et. al (2012) is one of the earlier researchers that introduced Kinect for human gait recognition with promising recognition rates. Their study developed a method for human gait recognition of nine subjects in lateral view. 11 sets of static features and two sets of dynamic features were generated from the skeleton joints that were extracted from Kinect. The feature sets were classified using Naïve Bayes, One Rule (1R) and C4.5 algorithms with 91\% recognition accuracy using four static gait features and Naïve Bayes as classifier. The four static gait features were height, length of legs, length of torso and length of the left upper arm. In the same year, 2012, Naresh et al. (2012) also performed human gait recognition with Kinect. The researchers performed the recognition of 20 subjects in frontal view. 21 sets of static features were extracted and further classified by covariance analysis. Results showed that combined features contributed higher recognition accuracy as opposed to those of single features. The highest recognition accuracy attained was $93.33 \%$ by fusing gait features from the spine, the right arm, and the left leg. The result also showed that Kinect is a robust device with regards to multi-view scenarios.

Subsequently, in 2013, another study was conducted by Araujo et al. (Araujo, Graña, and Andersson 2013) and in this study they reported that features extracted through anthropometric identification using Kinect was a success as well. They investigated types of static gait features, classifiers and pose. The researchers focused on the gait of eight subjects in lateral and frontal views. The results revealed that the combination of the entire static gait features with $\mathrm{KNN}$ as classifier produced the highest recognition accuracy of $99.6 \%$. The next research on human gait recognition with Kinect was carried out as reported in (Gianaria et al. 2013). In this research, the gait recognition of 20 subjects in frontal view was performed. The study extracted various sets of static features and dynamic features within one gait cycle in order to obtain the most significant features for gait recognition purposes and K-means unsupervised algorithm with Euclidean distance used for classification. As a result, dynamic features, specifically the combination of distance between knees, distance between elbows, movement of head in the $\mathrm{x}$-coordinate and movement of head in the $y$-coordinate were found to be the most significant features with highest recognition accuracy of $52 \%$. A year later, the same researchers further explored and investigated both static and dynamic gait features of twenty subjects (Gianaria et al. 2014). This time, they revealed that movement of elbows, knees, and head were significant features in recognizing 
human gait. With SVM as classifier, the recognition of human gait with 20 subjects showed $96.25 \%$ accuracy rate.

As discussed in Sadhu and Konar (2014), nine from the 20 skeleton joints of 25 subjects were employed. The nine features include hip centre, left and right elbow, left and right hand, left and right knee along with left and right foot. The hip centre was used for normalization of all eight skeleton joints. The features were generated from all eight joints. The study proposed recognition using differences between total feature error and standard deviation. As a benchmark, SVM and KNN were implemented as classifiers and the results were then compared. The proposed method resulted accuracy recognition rate of $92.48 \%$. Conversely, another investigation by Andersson and Araújo (2014) on a 3D skeleton data with a multi-view-angle imagery was also done using Kinect. In this study the lower limbs of one hundred and sixty-six subjects were extracted and analysed. Potential features of skeleton joints such as kinematic features and anthropometry features within one gait cycle were extracted as well. The Multilayer Perceptron (MLP) with 20 hidden numbers and KNN were used to classify the features. Interestingly, the results showed that the highest recognition accuracy was acquired with the anthropometry features used as input features to the classifier. It was also observed that the recognition accuracy decreases as the number of subject increases from 10 to 160 . Furthermore, investigation on the frame number was carried out and it was found that higher number of gait frames contributed to higher recognition accuracy with KNN as classifier and anthropometry features as inputs.

In addition, the recognition of 10 subjects walking in lateral view was carried out by Jiang et. al (2014). Two feature sets were generated; static and dynamic feature sets within one full gait cycle from certain skeleton joint points in 3D space. Dynamic time warping (DTW) is then used to normalize time frames for train and test datasets. As a result, $82 \%$ recognition accuracy was obtained using both static and dynamic features with neural network (NN) as classifier. Furthermore, a research on multi-view angle using Kinectbased human gait analysis was performed by Cheewakidakarn and Nattee (2014). In this research, videos of 17 walking subjects in various angle views such as lateral, frontal and oblique views were recorded. Seventeen sets of static gait features and three sets of dynamic features were extracted. DTW was employed as pattern classifier. It was reported that $51.77 \%$ of accuracy was achieved with fusion of static and kinematic as gait features.

\section{OPTIMISATION OF GAIT FEATURE EXTRACTION USING KINECT}

A year after Kinect was introduced for human gait recognition, studies on extracting potential gait features have been expanded for optimisation or selection of the extracted features. Sinha et al. (2013) employed ANN as feature selection in recognition of ten subjects; walking in lateral view. Two sets of features namely static features and hybrid features within each half gait cycle were extracted from skeleton joints and classified using ANN. The results reported that the static features were the most significant features compared to the dynamic features with accuracy rate of $62 \%$ obtained with hybrid features and ANN as feature selection. Additionally, study by Sinha et al. reported better recognition rate as compared to the studies by Preis et al. (2012) and Ball et al. (2012).

Progressive researches on feature optimisation in human gait recognition were carried out continuously in 2014 . Dikovski et. al (2014) employed PCA as a feature selection technique in their work. In this study, seven potential feature sets were generated from specific skeleton joints extracted from 15 subjects at lateral view using Kinect. The gait features were extracted within one full gait cycle. Next, MLP, SVM and $\mathrm{J} 48$ decision tree algorithm were chosen to evaluate the effectiveness of the gait features for recognition purposes. As a result, the highest recognition accuracy was obtained with MLP classifier based on features with joint distance. Besides that, features of lower body attained higher accuracy as compared to the upper body since most of the upper parts were hidden whilst the worst classification accuracy obtained was using PCA as feature extraction.

Another study that reported poor recognition accuracy using PCA as a feature selection is the work by Molina et al. (2014). In this study, recognition of ten subjects walking in lateral, oblique and frontal movements in front of the Kinect were performed using WEKA application. The results attained showed that low recognition accuracy of $70 \%$ obtained with feature selections via PCA as opposed to the original features that attained higher recognition accuracy specifically of $90 \%$. The results also proven that the most significant features were fusion of seven features namely the length of the right and left shoulder, height, the length of the right arm, the length of the right knee and right foot, and the length of the right hip. Also, in 2015, Ahmed et al. (2015) proposed another feature extraction and feature optimisation method. In this proposed method, vector of the joint angle between two skeleton joints with respect to the spine joint is extracted as features. The features were extracted within a full gait cycle. The most significant feature was selected based on large variation of gait features while walking, whereas features with smaller variations were eliminated. For recognition stage, the study used DTW-kernel. As a result, the proposed method obtained a $93.30 \%$ recognition rate as compared to the results in the study obtained in Preis et al. (2012).

Another feature optimisation method was proposed by Prathap and Sakkara (2015). The researchers extracted static features such as height and the length of both legs and both hands. In addition, they proposed dynamic features such as the angle between the left hand and hip centre, and the angle between the right hand and hip centre. This research also extorted other dynamic features such as step length and distance between centroids. The features were extracted from the entire sequences of frames. For the optimisation process, the extracted features were then further chosen at a single value. The selected single features were chosen based on the traits they possess. For example, for the height attribute, the maximum value is chosen as the selected feature and 
standard deviation for the hand angle. The optimized features were then fed to classifiers such as Levenberg-Marquardt back propagation and correlation algorithm. The end result indicated that features such as height, distance between centroids and step length proven to be significant features in recognition of the five subjects. To sum it up, LevenbergMarquardt back propagation excelled at an accuracy of $94 \%$.

\section{THEORETICAL BACKGROUND}

\section{PRINCIPAL COMPONENT ANALYSIS (PCA)}

The Principal Component Analysis (PCA) is a well-known feature reduction method employed in pattern recognition area. PCA is mainly used to select useful information and remove the superfluous information as well. The information said here is referred to as features. Firstly, PCA transforms features into the orthogonal linear space, known as principal components (PCs) and calculates their variances. Then, the PCs are ranked according to their variances. $\mathrm{PC}$ with the largest variance is ranked at the top and vice versa.

The critical step is to eliminate the redundancy and insignificant features. Originally, there are three methods namely cumulative percentage of variance, eigenvalueone-criterion and scree test. In the cumulative percentage of variance method, features with variances accumulated up to $80 \%$ are retained and the remainders are eliminated. In the eigenvalue-one-criterion and the scree test methods, features with eigenvalue less than one and features with large variation are eliminated respectively. According to previous research, employment and performance of the selection methods of PCA varies with the respective research area. In cases like classification of infant cry (Sahak et al. 2011), the eigenvalue-one-criterion excelled than the other two methods, however in other cases such as the texture of SAR image (Chamundeeswari \& Singh 2009), the SCREE test was selected as the best method.

\section{LOCALLY LINEAR EMBEDDED (LLE)}

To begin with, Locally Linear Embedded (LLE) is suitable for identifying significant features, to reduce dimensionality of the input features as well as to enhance recognition performances. Unlike PCA, LLE is designed for nonlinear dimensionality reduction of high dimensionality features. Let the input matrix $X$ in the size of $D$ by $N$. LLE produces an output of $Y$ in the size of $d$ by $N$ where $d<<D$. Note that the $K$-th column vector of $Y$ corresponds to the $K$-th column of vector $X$. Basically, there are three main processes in LLE. Firstly, $K$, known as the nearest neighbour is computed for each feature point, using Euclidean distances. Then, reconstruction errors resulting from the approximation of each feature point, $X_{i}$ by its $K$ are measured by the cost function as shown in Equation 1:

$$
\varepsilon(W)=\sum_{i=1}^{N}\left\|X_{i}-\sum_{j=1}^{K} W_{i j} X_{j}\right\|^{2}
$$

At the same time, the reconstruction weights $W_{i j}$ are computed to minimize minimise (1), subject to two constraints:

1. Each feature point $X_{i}$ is reconstructed only from its neighbours, enforcing $W_{i j}=0$ if $X_{j}$ if does not belong to this set.

2. The rows of the weight matrix sum to one:

$$
\sum_{j=1}^{K} W i_{j}=1
$$

Finally, the low dimensional embedding is computed where each high dimensional observation $\vec{X}_{i}$ is mapped to a low dimensional vector $\vec{Y}_{i}$. This is done by fixing the weight and minimizing minimising a cost function as shown in Equation 2:

$$
\Phi(Y)=\sum_{i=1}^{N}\left\|Y_{i}-\sum_{j=1}^{K} W i_{j} Y_{j}\right\|^{2}
$$

under constraints $\frac{1}{N} \sum_{i=1}^{N} Y_{i} Y_{i}{ }^{T}=I$ (normalized unit covariance) and $\sum_{i=1}^{N} Y_{i}=0$ (translation-invariant embedding), which provide a unique solution.

In order to find matrix $Y$ under these constraints, a new matrix is constructed based on matrix $\mathrm{W}$ as shown in Equation 3:

$$
M=(1-W)^{T}(1-W)
$$

Next, LLE computes bottom $d+1$ eigenvectors of $M$, associated with the $d+1$ smallest eigenvalues. The first eigenvector (composed of 1's) by which the eigenvalue is close to zero is excluded. The remaining $d$ eigenvectors yield the final embedding of $Y$ (Kouropteva and Pietik 2003). A good LLE mapping is based on two parameters that need to be adjusted; the dimensionality $d$ to be mapped to and the number of neighbours $K$.

\section{MULTI-CLASS SVM}

Originally SVM is designated for binary class problem. Further, the SVM is used to solve multi-class problems. Numerous binary classifiers were constructed and their performances were computed. The performances were compiled to gauge as overall recognition performance. Several approaches were introduced in the designation of multi-class SVM, such as oneversus-one (OVO), one-versus-all (OVA) and error correcting output code (ECOC).

Among these approaches, ECOC is proven capable to solve classification of three and more classes by reducing bias and variances that were introduced in the learning algorithm (Escalera 2010; Escalera \& Pujol 2006). ECOC can be divided into two main components. The first component is coding design. In this component, base codeword for each trained binary classes were designed. The common used coding design is OVA. In OVA, the number of binary classifier is similar to the number of class. Let the number of class be known as L. Hence, there are L binary classifiers. For each binary classifier, one class is designated as positive class and the rest are designated as negative class. Therefore, the L codeword is then generated from the trained binary classifiers. 
The L codeword is then decoded in the second component, which is known as decoding scheme. This scheme determines the predictions of the binary classifiers to be combined. Here, a new codeword is generated from the test data. Then the new codeword is compared with the base codeword that was obtained in the coding design. To compare the codeword, classification error is used to determine the performances of the classifiers. Similar to original SVM, the multi-class SVM also has kernels in solving problem of non-separable data. The common kernel used is radial basis kernel (RBF). The optimal SVM model can be obtained by selecting appropriate regularization parameter $(C)$ and sigma $(\sigma)$ as well.

\section{METHODOLOGY}

The general overview of the proposed methodology for human gait recognition using Kinect is as depicted in Figure 1. Firstly, the database of each subjects is recorded in the experimental area as per depicted in Figure 2. Next, all database need to go through the pre-processing stage. Upon completion of pre-processing, the most vital stage for gait recognition is the feature extraction. For this feature extraction stage, the original gait features as well as feature selection using PCA and LLE will be evaluated. Next is the classification of each subject using SVM classifier followed by evaluating the robustness of the developed method based on performance measures.

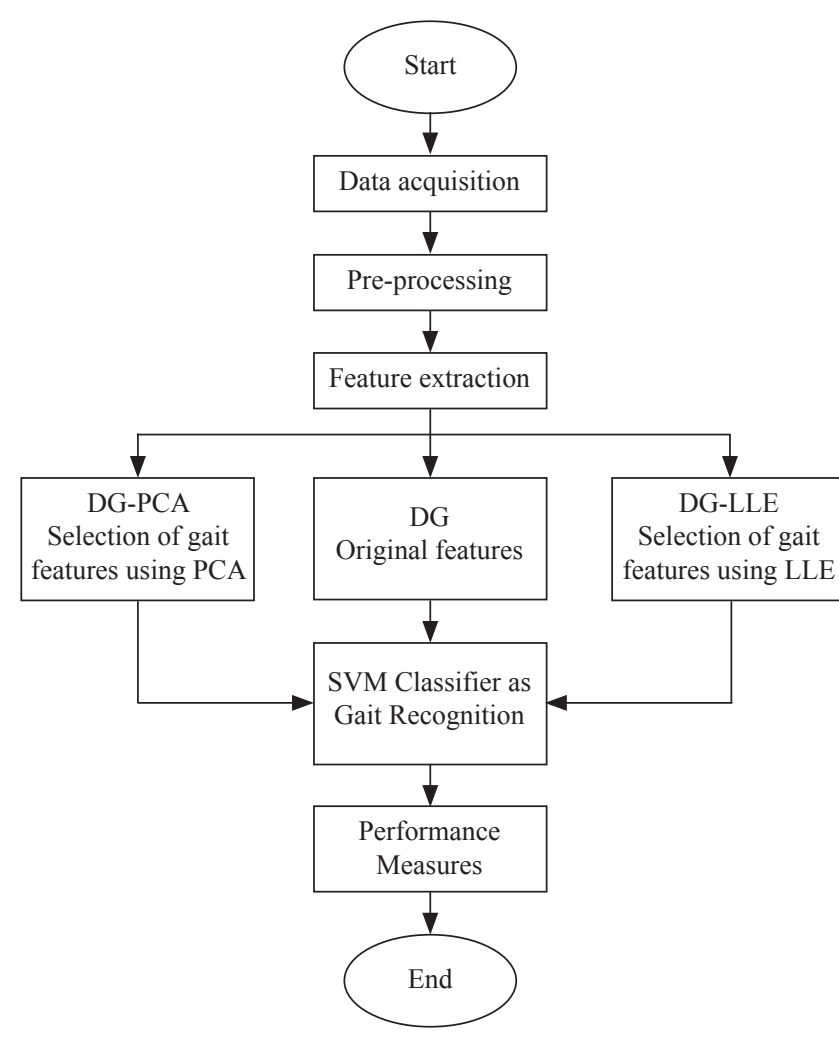

FIGURE 1. Overall process in the recognition of human gait using Kinect

\section{DATA ACQUISITION}

As mentioned earlier, this study investigated the gait features of 30 subjects in an indoor environment. The data acquisition stage was performed in daylight, in a laboratory. Figure 2 and Figure 3 show the layout of the experimental area. Here, the Kinect was placed 1 meter above the floor, as illustrated in Figure 2. The red area indicates the covered captured area, and this is the area of walking gait of subjects is recorded. Firstly, 11 male subjects and 19 female subjects were required to perform their normal walking gait using their normal speed. In order to gain normal walking patterns, the subjects began walking outside of the captured area. The subjects were requested to walk repeatedly for 10 times and no restriction on clothing types except for pants. In addition, each of the subject's information such as age, height and weight were recorded as well.

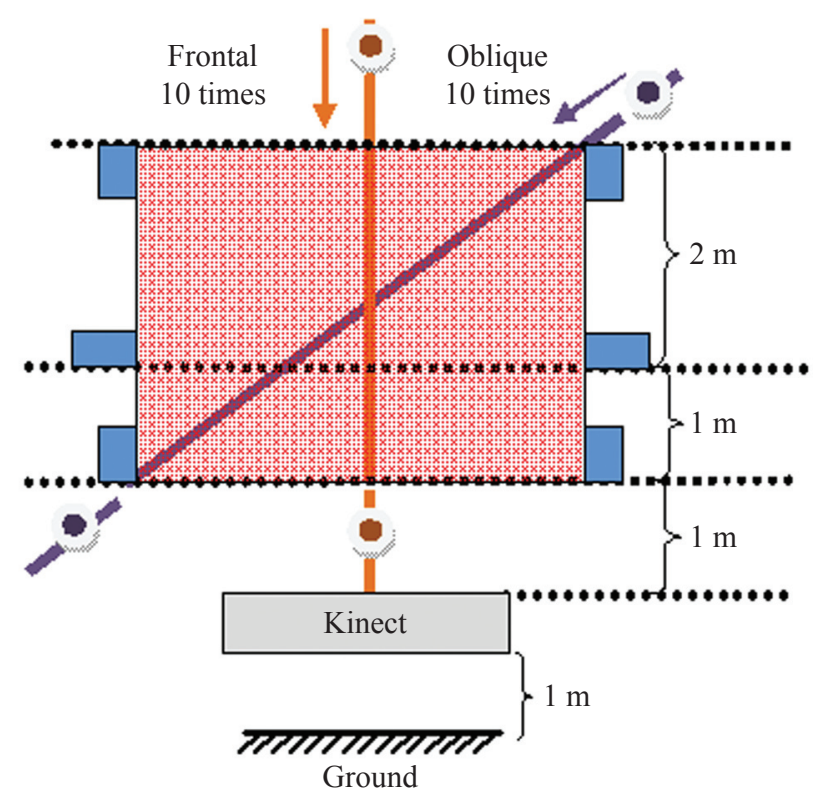

FIGURE 2. Top view of the walking gait acquisition experimental area

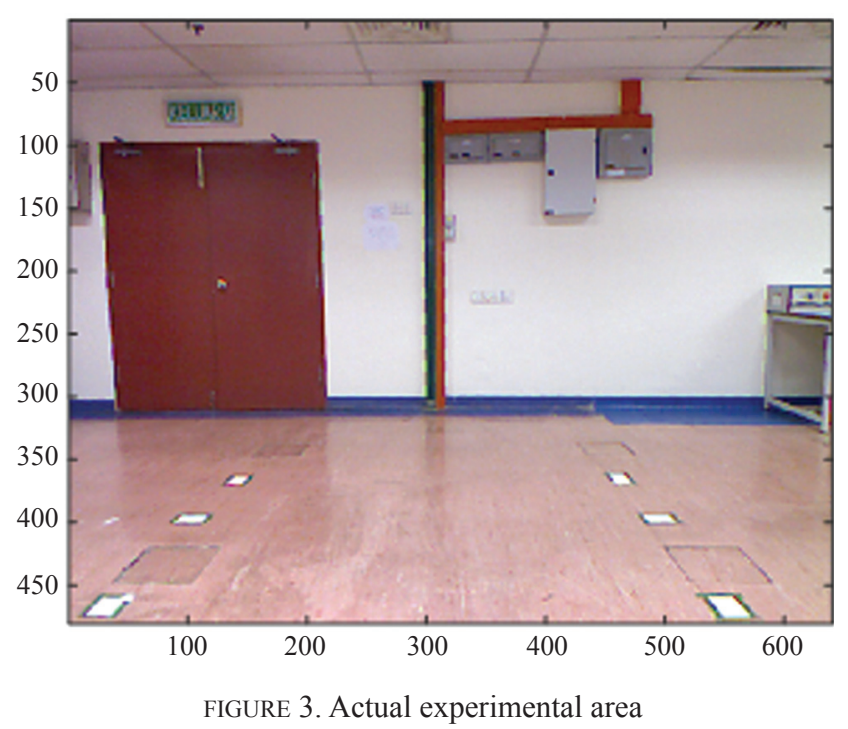


The main aim of this stage is to normalise skeleton joints, considering that the skeleton image increases as the subject walks towards the Kinect. Firstly, empty frames resulted from the recorded skeleton data were removed prior to normalization of the skeleton joints. The empty frames are due to extra frames obtained at the beginning and end during recording of the walking gait of each subject.

The normalisation was performed in xyz-coordinate. At first, the skeleton joint points are standardised at the height reference, $h_{r}$ or $i$-th frame using (4). For each subject, the height at half of the trimmed frame was represented as the reference height.

$$
j_{(\text {new)i }}=\left(j_{(o l d) i} \div h_{i}\right) \times h_{r}
$$

where $j_{\text {old }}$ is the original skeleton joint, $j_{\text {new }}$ is the new skeleton joint, $h$ is the height and $h_{r}$ is the height reference.

The relative movement of the standardised skeleton joints was then scaled at a constant head joint. The purpose of this step is to replicate the subjects' walk on a treadmill The head joint was fixed at one point using (5).

$$
j h_{(f x) i}=j h_{r}-j h_{(n e w) i}
$$

where $j h_{r}$ is the reference head joint and $j h_{\text {new }}$ is the new head joint.

The normalized skeleton joints were then calculated using (6)

$$
j_{(n o r m) i}=j h_{(n e w) i}+h j_{(f x) i}
$$

The pre-processed skeleton joints were further extracted in the next stage.

\section{FEATURE EXTRACTION}

In this research, skeleton joints in the xyz-coordinates; also known as skeleton joint points were extracted within one full gait cycle. Figure 4 showed the steps involved in the feature extraction stage. Firstly, detection of full gait cycle upon completion of pre-processed skeleton joint points was performed. Note that the resulted gait cycles consist of various numbers of frames which can lead to difficulty in the classification stage. Hence, synchronization process was implemented in order to standardize the number of frames. Upon standardisation, the skeleton joint points were extracted as input features.

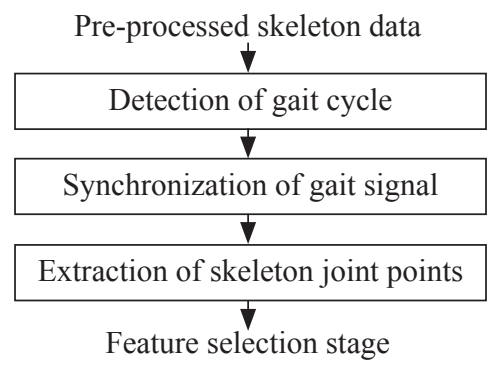

FIGURE 4. Feature extraction stage of human gait recognition using kinect
Gait cycle is a period of a sequence of the same foot touching the ground twice (Boulgouris \& Plataniotis 2005; Sinha et al. 2014). Thus, the gait cycle was computed based on the distance between the right ankle and left ankle. Throughout this study, distance between the right ankle and left ankle in the xy-coordinates was calculated for oblique view, while for frontal view, the distance between the right ankle and left ankle was computed in xyz-coordinates. In addition, the distance vector was filtered and smoothed using SavitzkyGolay moving average algorithm. Local minima; which is transition from negative slope to positive slope is detected from the distance vector and one full gait cycle comprised of three consecutive local minima (Dikovski \& Gjorgjevikj 2014; Sinha \& Bhowmick 2013). For simplicity, the detection of gait cycle is illustrated in lateral view, as depicted in Figure 5. Therefore, one to three gait cycles were computed for oblique view whilst for frontal view, one to two gait cycles were computed. For standardization purposes, only gait features within one gait cycle for each walking sequence per subject is used for further analysis. Since there are 30 subjects and 10 walking sequence, thus the gait features were made of 300 gait cycles for both oblique and frontal view. For the next section, these 300 gait cycles were named as 300 samples.

\section{SYNCHRONIZATION OF GAIT FEATURE}

For each subject, the number of frames extracted for each gait cycle is dissimilar for oblique and frontal views. For instance, 12 frames to 26 frames were attained for the resulted gait cycles for both the oblique and frontal views. This is due to the different walking speed amongst the subjects since they are allowed to walk at their normal comfortable speed. Hence, the diverse numbers of frames could cause unequal size of feature patterns, which can lead to intricacy in the classification stage. As such, the synchronisation stage of gait features was implemented in order to standardise the size of the input feature via spline interpolation process that synchronised the number of frame for each gait cycle to a similar size. Spline interpolation is a mathematical algorithm commonly used for curve fitting (Spline Curves 2016). It is a process of generating new data points within the range of a discrete set of original data points and smoothing the set of data points as well. Consequently, gait cycles with the size of maximum numbers of frames, 26 frames were fixed for both frontal and oblique views.

\section{EXTRACTION OF SKELETON JOINT POINTS}

As mentioned earlier, this study is concerned with the dynamic gait features, known as skeleton joints. Thus, in this study, skeleton joints in the xyz-coordinates were designated as skeleton joint points. Kinect provides 20 skeleton joints in the xyz-axis. Hence, a total of 60 skeleton joint points were extracted in this stage. Firstly, skeleton joint points for each subject were extracted in a column vector using (7). Here 


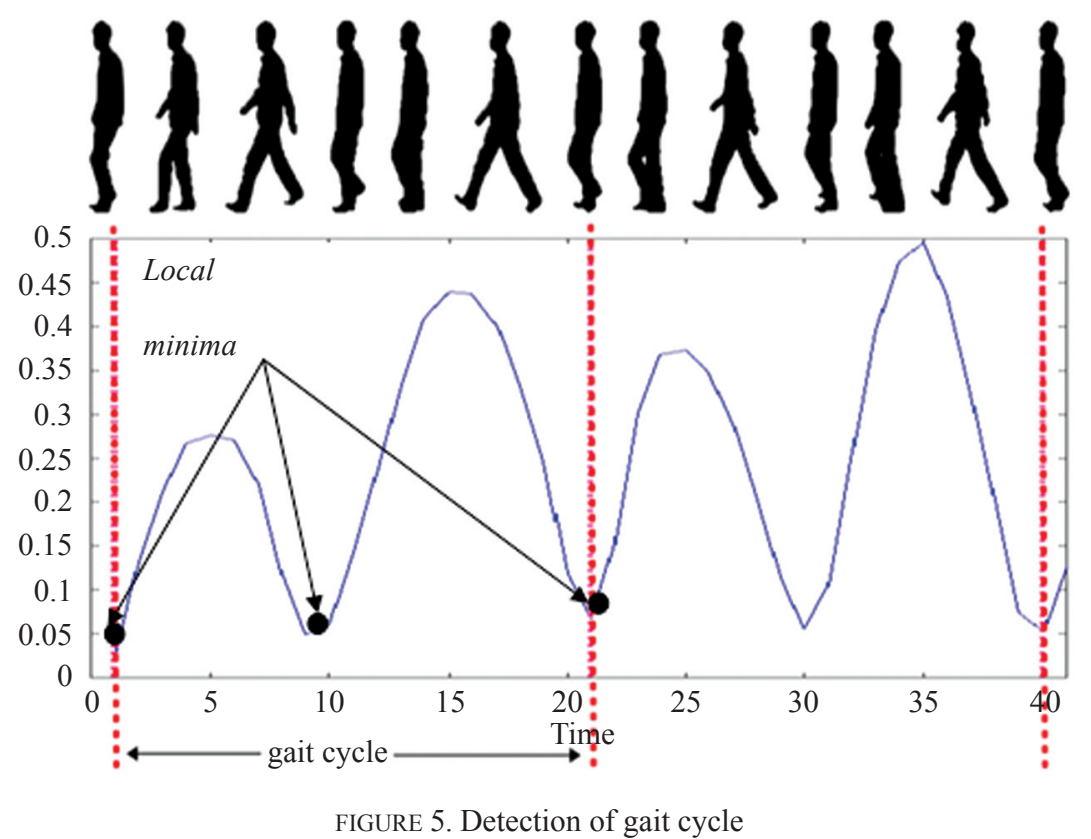

the 20 skeleton joints in the $\mathrm{x}$-coordinate were arranged followed by the same 20 skeleton joints in the $y$-coordinate and finally the skeleton joints in the z-coordinate. As a result, the extracted gait feature size was 60 by 26 .

$$
j_{x n} j_{y n} j_{z n} \times i
$$

where $j_{x}$ is the skeleton joint in $\mathrm{x}$-coordinate, $j_{y}$ is the skeleton joint in $y$-coordinate, $j_{z}$ is the skeleton joint in $\mathrm{z}$-coordinate, $i$ is the number of frame and $n$ is the number of skeleton joint.

To arrange these as input features as obtained in (7), a column vector format was used for all 30 subjects. As previously mentioned, 10 samples were allocated for each subject, this resulted a 1560 by 300 feature sizes. Figure 6 illustrates the 20 skeleton joints and their designated label, provided by the Kinect.

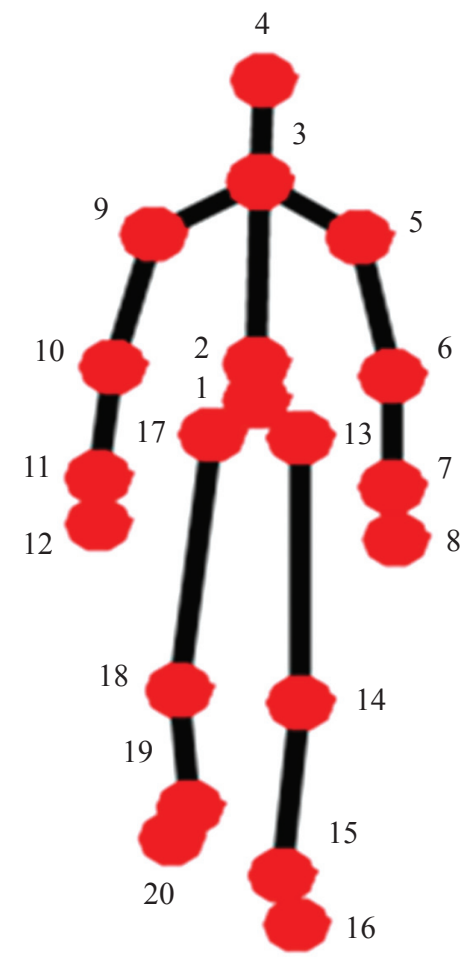

\begin{tabular}{cl}
\hline Number of Skeleton Joint & Name of Skeleton Joints \\
\hline 1 & Hip_center \\
2 & Spine \\
3 & Shoulder_center \\
4 & Head_ \\
5 & Shoulder_left \\
6 & Elbow_left \\
7 & Wrist_left \\
8 & Han__left \\
9 & Shoulder_right \\
10 & Elbow_right \\
11 & Wrist_right \\
12 & Hand_right \\
13 & Hip_left \\
14 & Knee_left \\
15 & Ankle_left \\
16 & Foot_left \\
17 & Hip_right \\
18 & Knee_right \\
19 & Ankle_right \\
20 & Foot_right \\
&
\end{tabular}

FIGURE 6. Skeleton joints provided by Kinect 


\section{PATTERN RECOGNITION}

In this stage, the obtained input features were first normalised with zero mean and unity standard deviation. Then, the input features were mapped in the range of -1 to +1 prior to inputs of the classifier. In this study, RBF kernel SVM was chosen due to its good performance in the pattern recognition area (Cheng \& Xu 2007; Kusy 2004; Sahay et al. 2016; Hall 2015; Yan \& Wang 2009; Banerjee et al. 2015; Purnama et al. 2015; Meddeb \& Karray 2015). In the selection of the optimal model of SVM classifier with RBF kernel, both $C$ and $\sigma$ varies from 10 to 100 with increment of 10 . In order to determine the best optimisation method, three approaches were evaluated and tested. The three approaches are described as follows;

\section{ORIGINAL GAIT FEATURES (DG)}

In the first approach known as DG, gait features were extracted directly from the feature extraction stage and the original gait features were the inputs to the SVM classifier with RBF as kernel.

FEATURE EXTRACTION VIA PCA ( DG-PCA)

In the second approach, the gait features were selected by PCA as feature selection prior to classification stage using SVM with RBF kernel. This approach was designated as DGPCA. The input features were transposed as 300 by 1560 before projected into a high dimensional space and variances computed, which further resulted the principal components (PCs). Then, the PCs with the largest variances were retained whilst PCs with smallest variances were discarded. In this study, PC with the cumulative variance of $99 \%$ was retained.

\section{FEATURE EXTRACTION VIA LLE (DG-LLE)}

In the third approach, the gait features were selected using LLE before acting as input features to the SVM classifier and RBF as kernel. This approach was designated as DGLLE. With LLE employed, firstly $K$, the nearest neighbour is computed for each input feature using Euclidean distances. Then, cost function was calculated using (1) in determining the reconstruction errors from the estimation of the input feature by its $K$. The input feature was then mapped to a low dimensional embedding feature using (2). An investigation into the number of neighbours, $K$ and the dimensionality of low embedding feature, $d$ was performed in order to determine the most significant gait features as well as the optimal LLE mapping. To attain the significant gait features, $K$ was tuned from 84 to 100 with increment of 4 and $d$ was varied from 50 to $K-2$ with increment of 2 .

\section{RECOGNITION PERFORMANCE}

Performances of human gait recognition such as recognition accuracy, specificity and sensitivity were evaluated in order to gauge effectiveness of the proposed approach (Hossin \& Sulaiman 2015; Sokolova \& Lapalme 2009). Correct recognition rate (CRR) is the average of correct recognition of all subjects using 10-fold cross validation approach. The correct recognition is calculated as follows:

$$
\mathrm{CRR}=\frac{\sum_{i=1}^{l} \frac{t p+t n}{n}}{n}
$$

where $l$ is the number of subject, $t p$ is the true positive, $t n$ is the true negative, $n$ is the number of sample.

Sensitivity is evaluated to measure the fraction of an appointed subject that is correctly classified. It can be calculated by:

$$
\text { Sensitivity }=\frac{\sum_{i=1}^{l} \frac{t p}{t p+f n}}{l}
$$

Specificity is used to calculate the fraction of nonappointed subject that is correctly classified.

$$
\text { Specificity }=\frac{\sum_{i=1}^{l} \frac{t n}{t n+f p}}{l}
$$

\section{RESULTS AND DISCUSSIONS}

In this section the conducted experiment and results attained are elaborated and discussed. It was computed that the average subjects aged 30.10 years old have the average height and weight of $1.60 \mathrm{~m}$ and $61.68 \mathrm{~kg}$ respectively.

\section{OPTIMISATION OF SVM MODEL USING RBF KERNEL}

Firstly, the value of $C$ and $\sigma$ as of all three methods are tabulated in Table 1. As mentioned earlier, selection of optimal parameter for RBF kernel was performed by varying $C$ and $\sigma$ from 10 to 100 , with an interval of 10 . In the case of DG, for both the frontal and oblique views, large $C$ and $\sigma$ were required in determining the optimal RBF kernel for the SVM model. It was observed that the least value of $C$ and $\sigma$ were required for DG-PCA, for both oblique and frontal views as well. As for DG-LLE, the value for $\sigma$ was similar as DG-PCA however the value of regularization parameter $C$ was the highest amongst the three methods for frontal view in order to construct the optimal classifier. With these selections of regularization; parameter $C$, RBF kernel value $\sigma$, and all three methods attained $100 \%$ CRR for the training datasets.

TABLE 1 . The optimal $C$ and $\sigma$ for DG, DG-PCA and DG-LLE with SVM as classifier

\begin{tabular}{ccccc}
\hline No. & View & Approach & C & $\sigma$ \\
\hline \multirow{3}{*}{ 1. } & \multirow{2}{*}{ Frontal } & DG & 80 & 90 \\
& & DG-PCA & 20 & 20 \\
& & DG-LLE & 90 & 20 \\
2. & Oblique & DG & 80 & 90 \\
& & DG-PCA & 30 & 10 \\
& & & 50 & 10 \\
\hline
\end{tabular}


FEATURE SELECTION OF GAIT FEATURES USING PCA

The selections of input features using PCA algorithms such as eigenvalue-one-criterion, cumulative percentage of variance, and scree test were reported in this section. For the cumulative percentage of variances; in order to obtain significant features, variances accumulated from $80 \%$ to $99 \%$ were computed. Upon completion of experimental analysis, cumulative percentage of variance of $99 \%$ was chosen as the best selection method since poor recognition accuracy was obtained for the variance accumulated less than $99 \%$ and in the other two selection methods as well. Therefore, only results from the cumulative percentage of variance of $99 \%$ were presented. Table 2 tabulates the first 68 PCs with their cumulative variance (\%) for frontal and oblique views. The shaded area indicates PCs that were selected and retained. The PCs were retained until variance of $99 \%$ was achieved. Hence, in this analysis, 66 PCs were retained for frontal view and 23 PCs for oblique view. As a result, the dimension of the input features for DG-PCA was reduced from 1560 by 300 to 66 by 300 and 23 by 300 for frontal and oblique view, respectively.

TABLE 2. Cumulative variance (\%) for the first 68 PCs for frontal and oblique view

\begin{tabular}{|c|c|c|c|c|c|}
\hline $\mathrm{PC}$ & Frontal & Oblique & $\mathrm{PC}$ & Frontal & Oblique \\
\hline 1 & 37.281 & 69.795 & 35 & 98.039 & 99.289 \\
\hline 2 & 70.376 & 86.600 & 36 & 98.092 & 99.308 \\
\hline 3 & 80.187 & 92.813 & 37 & 98.141 & 99.325 \\
\hline 4 & 87.614 & 96.873 & 38 & 98.186 & 99.342 \\
\hline 5 & 89.856 & 97.343 & 39 & 98.231 & 99.358 \\
\hline 6 & 91.377 & 97.630 & 40 & 98.273 & 99.374 \\
\hline 7 & 92.583 & 97.839 & 41 & 98.313 & 99.389 \\
\hline 8 & 93.338 & 97.981 & 42 & 98.352 & 99.404 \\
\hline 9 & 93.916 & 98.121 & 43 & 98.389 & 99.418 \\
\hline 10 & 94.425 & 98.231 & 44 & 98.426 & 99.432 \\
\hline 11 & 94.811 & 98.332 & 45 & 98.461 & 99.445 \\
\hline 12 & 95.152 & 98.424 & 46 & 98.495 & 99.457 \\
\hline 13 & 95.432 & 98.500 & 47 & 98.528 & 99.469 \\
\hline 14 & 95.692 & 98.575 & 48 & 98.559 & 99.481 \\
\hline 15 & 95.914 & 98.639 & 49 & 98.590 & 99.492 \\
\hline 16 & 96.122 & 98.696 & 50 & 98.619 & 99.503 \\
\hline 17 & 96.312 & 98.749 & 51 & 98.647 & 99.513 \\
\hline 18 & 96.471 & 98.799 & 52 & 98.675 & 99.524 \\
\hline 19 & 96.618 & 98.844 & 53 & 98.703 & 99.534 \\
\hline 20 & 96.755 & 98.885 & 54 & 98.730 & 99.543 \\
\hline 21 & 96.884 & 98.925 & 55 & 98.755 & 99.552 \\
\hline 22 & 97.010 & 98.962 & 56 & 98.780 & 99.561 \\
\hline 23 & 97.126 & 98.997 & 57 & 98.805 & 99.570 \\
\hline 24 & 97.232 & 99.028 & 58 & 98.828 & 99.579 \\
\hline 25 & 97.332 & 99.058 & 59 & 98.851 & 99.587 \\
\hline 26 & 97.422 & 99.086 & 60 & 98.874 & 99.595 \\
\hline 27 & 97.507 & 99.114 & 61 & 98.896 & 99.603 \\
\hline 28 & 97.589 & 99.139 & 62 & 98.918 & 99.611 \\
\hline 29 & 97.663 & 99.163 & 63 & 98.938 & 99.619 \\
\hline 30 & 97.734 & 99.187 & 64 & 98.958 & 99.626 \\
\hline 31 & 97.803 & 99.209 & 65 & 98.977 & 99.633 \\
\hline 32 & 97.865 & 99.230 & 66 & 98.996 & 99.640 \\
\hline 33 & 97.925 & 99.250 & 67 & 99.015 & 99.647 \\
\hline 34 & 97.984 & 99.270 & 68 & 99.033 & 99.654 \\
\hline
\end{tabular}

OPTIMIZATION OF GAIT FEATURES USING LLE

The optimisation of gait features using LLE was performed via selection of suitable LLE parameter; namely the dimensionality $d$ was mapped and the number of neighbours $K$. Hence, $K$ and $d$ was varied as aforementioned in the previous section. The resulted $K$ and $d$ led to the variation of inputs features. The input features were inputs to the optimal model of SVM classifier. CRR on test data was computed and compared 
for each $K$ and $d$, so that the optimum input features can be determined. Consequently, for frontal view, the optimal input features attained were at $K=84$ and $d=64$ while for oblique view, the finest input features attained were at $K=100$ and $d$ $=98$ respectively. Figure 7 demonstrates the selection of the finest $K$ when $d$ was set at 98 and 64 for the oblique and frontal view, respectively. For oblique view, for $K=100$ the CRR attained was $94.67 \%$ whilst for frontal view, the CRR slowly fluctuated as $K$ varied. Note that highest CRR at $98.26 \%$ achieved at $K=84$. Figure 8 below depicts the selection of suitable $d$ when $K$ was fixed at 100 for the oblique view and as $K$ was set to 84 for the frontal view. As can be seen in the oblique view, the CRR oscillated over the valued of $d$. Highest CRR of $89.26 \%$ was attained at $d=68$. As for the frontal view, the highest CRR was at $98.26 \%$ for $d=64$.

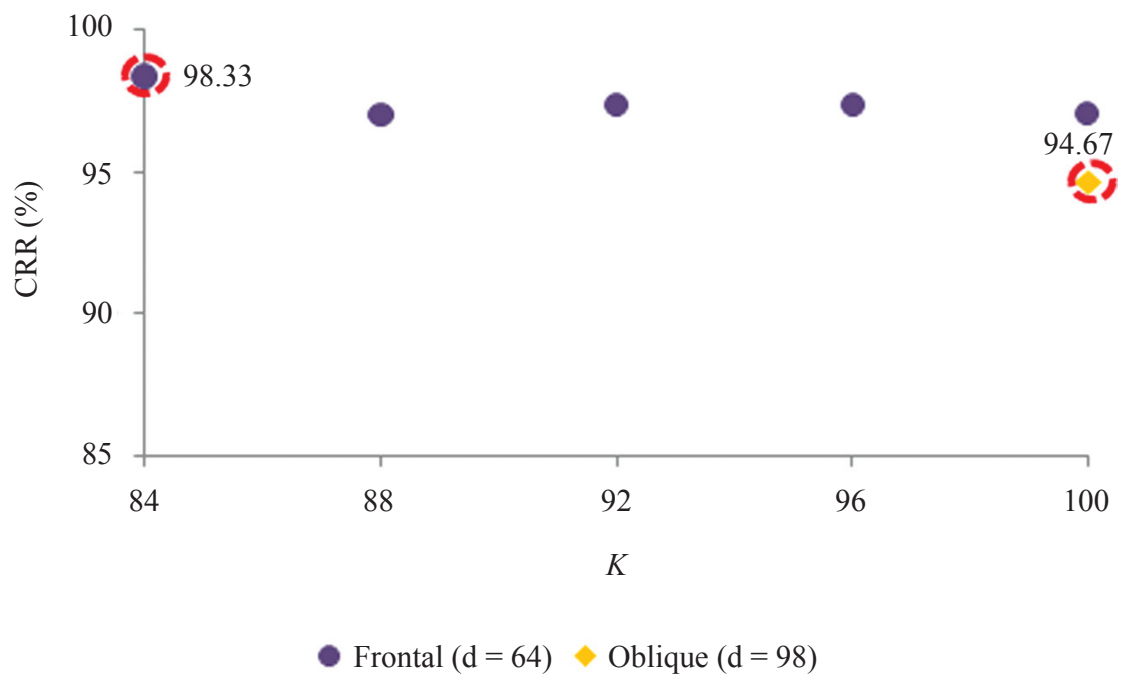

FIGURE 7. Results for selection of the best $K$, at $d=98$ (Oblique View) $\& d=64$ (Frontal View)

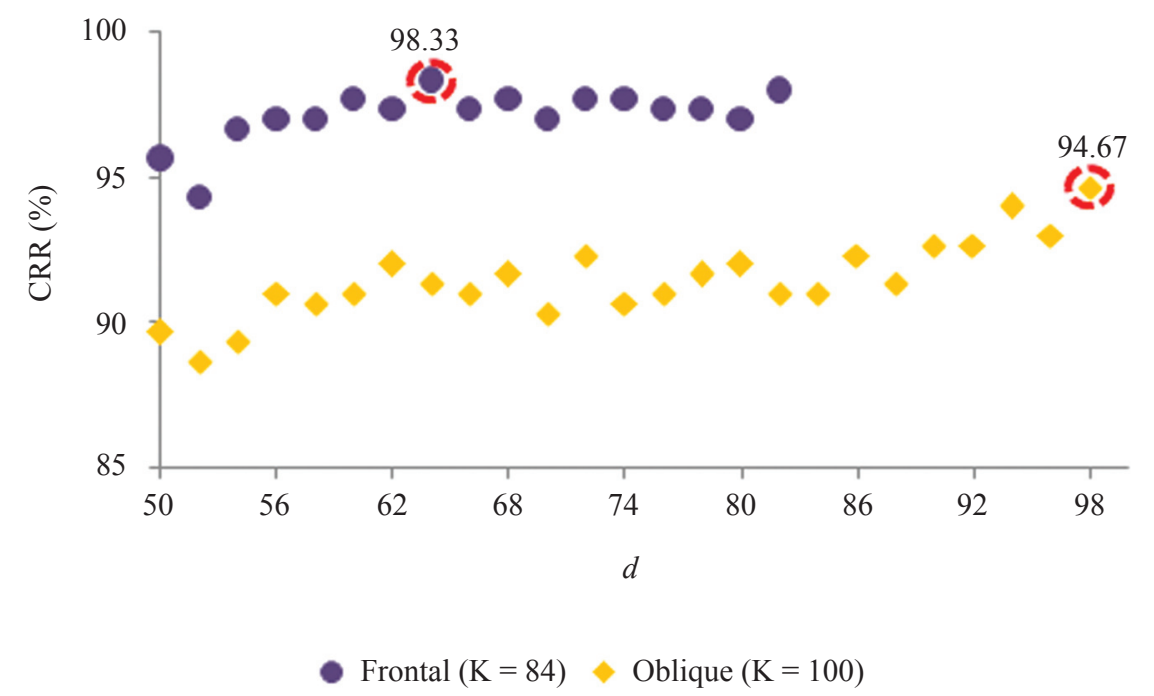

FIGURE 8. Results for selection of optimum $d$ at $K=100$ (Oblique View) $\& K=84$ (Frontal View)

\section{PERFORMANCE MEASURE}

The recognition performance for DG, DG-PCA and DG-LLE is as tabulated in Table 3. It is apparent that feature optimisation method reduced the dimension of input features. For the frontal view, lesser dimension was attained for DG-LLE, followed by DG-PCA. However, it is the opposite for oblique view. For oblique view, it can be concluded that DG-LLE outshined with high CRR (94.67\%) for input features of 50. Sensitivity and specificity were also the highest as presented in Table 4. Though DG-PCA offered fewer dimensions of input features than DG-LLE, the CRR was not encouraging since it had lower CRR. Similar for oblique view, DG-LLE once again outperformed accuracy rate in frontal view as compared to DG and DG-PCA. As tabulated in Table 3, highest CRR of 98.33\% with low number of features namely 64 input features attained based on DG-LLE.

Additionally, highest accuracy was obtained for both sensitivity (98.33\%) and specificity (99.94\%) too. Based on sensitivity and specificity attained, it was revealed that the 
recognition of non-appointed subject was slightly higher than the appointed subject. Overall, the proposed method was proven capable to enhance the recognition of human gait. Moreover, the utmost recognition rate is during frontal gait. Conversely, Table 4 tabulates the comparison between other studies on human gait recognition in frontal view using Kinect. As shown, the proposed study based on LLE along with SVM and larger numbers of subjects namely 30 subjects outperformed with recognition rate of $98.33 \%$ as compared to other studies.

TABLE 3. Recognition performance for DG, DG-PCA and DG-LLE for frontal view and oblique view

\begin{tabular}{llcccc}
\hline View & Approach & \# of feature & CRR (\%) & Sensitivity (\%) & Specificity (\%) \\
\hline \multirow{7}{*}{ Frontal } & DG & 1560 & 96.67 & 96.67 & 99.86 \\
& DG-PCA & 66 & 96.67 & 96.67 & 99.86 \\
& DG-LLE & 64 & 98.33 & 98.33 & 99.94 \\
& DG & 1560 & 87.00 & 87.00 & 99.50 \\
Oblique & DG-PCA & 23 & 81.00 & 81.00 & 99.34 \\
& DG-LLE & 50 & 94.67 & 94.67 & 99.82 \\
\hline
\end{tabular}

TABLE 4. Comparison between other studies on human gait recognition in frontal view using Kinect

\begin{tabular}{clcc}
\hline No. & \multicolumn{1}{c}{ Researcher } & Number of subjects & CRR (\%) \\
\hline 1. & Prathap et al. (Prathap and Sakkara 2015) & 5 & 94.00 \\
2. & Naresh Kumar (Naresh Kumar and Venkatesh Babu 2012) & 20 & 97.50 \\
3. & Elena (Gianaria et al. 2014) & 20 & 96.00 \\
4. & Proposed study (Rohilah et al.) & 30 & 98.33 \\
\hline
\end{tabular}

\section{CONCLUSION}

This study investigated the optimisation of gait patterns using LLE and multi-class SVM in recognition of human gait. Results attained showed that DG-LLE outperformed for both oblique and frontal views for all three performance measures namely recognition rate, sensitivity as well as specificity. Moreover, the results also determined that the recognition of human gait in the frontal view excelled than that of the oblique view. Future work includes pre-processing of multiviews walking gait and extraction of these multi-views gait features to be evaluated for recognition purpose. Also, further investigation on other feature optimisation methods namely statistical analysis and other pattern classifiers specifically deep learning neural network and extreme learning machine for recognition human gait will be performed in the upcoming research projects.

\section{ACKNOWLEDGMENT}

This research is funded by Ministry of Higher Education (MOHE) Malaysia under the Niche Research Grant Scheme (NRGS) Project No: 600-RMI/NRGS 5/3 (8/2013) and Institute of Research Management and Innovation (IRMI), UiTM. The author thanked MOHE for the scholarship awarded under MyBrain MyPhD. The authors wish to thank Human Motion Gait Analysis (HMGA) Laboratory, IRMI Premier Laboratory, IRMI, UiTM, Malaysia for the instrumentation and experimental facilities provided as well as Faculty of
Electrical Engineering, UiTM Shah Alam for all the support given during this research.

\section{REFERENCES}

Ahmed, F., Polash Paul, P. \& Gavrilova, M.L. 2015. Kinectbased gait recognition using sequences of the most relevant joint relative angles. Journal of WSCG 23(2): 147-156.

Andersson, V., Dutra, R. \& Araújo, R. 2014, March. Anthropometric and human gait identification using skeleton data from kinect sensor. In Proceedings of the 29th Annual ACM Symposium on Applied Computing, 50-61.

Araujo, R.M., Graña, G. \& Andersson, V., 2013, March. Towards skeleton biometric identification using the microsoft kinect sensor. In Proceedings of the $28^{\text {th }}$ Annual ACM Symposium on Applied Computing, 21-26.

Ball, A., Rye, D., Ramos, F. \& Velonaki, M. 2012, March. Unsupervised clustering of people from 'skeleton' data. In Proceedings of the seventh annual ACM/IEEE international conference on Human-Robot Interaction, 225-226.

Banerjee, A., Pal, M., Tibarewala, D.N. \& Konar, A. 2015. Electrooculogram based blink detection to limit the risk of eye dystonia. In Advances in Pattern Recognition (ICAPR), 2015 Eighth International Conference on IEEE, 1-6. 
Boulgouris, N.V., Hatzinakos, D. \& Plataniotis, K.N. 2005. Gait recognition: a challenging signal processing technology for biometric identification. IEEE Signal Processing Magazine 22(6): 78-90.

Chamundeeswari, V.V., Singh, D. \& Singh, K. 2009. An analysis of texture measures in PCA-based unsupervised classification of SAR images. IEEE Geoscience and Remote Sensing Letters 6(2): 214-218.

Chang, J.Y. \& Nam, S.W., 2013. Fast random-forest-based human pose estimation using a multi-scale and cascade approach. ETRI Journal 35(6): 949-959.

Cheewakidakarn, A., Khamsemanan, N. \& Nattee, C. 2013. View independent human identification by gait analysis using skeletal data and dynamic time warping. In $14^{\text {th }}$ International Symposium on Advanced Intelligent Systems, 1-6.

Cheng, C.G., Cheng, L.Y. \& Xu, R.S. 2007. Classification of FTIR gastric cancer data using wavelets and SVM. In Natural Computation, 2007: Third International Conference on IEEE, 543-547.

Mei, F.C.C., Reaz, M.I., Leng, T.A. \& Yasin, F.M. 2007. Hardware prototyping of Iris recognition system: A Neural Network Approach. Jurnal Kejuruteraan 19: 77-86.

Dikovski, B., Madjarov, G. \& Gjorgjevikj, D. 2014. Evaluation of different feature sets for gait recognition using skeletal data from Kinect. In Information and Communication Technology, Electronics and Microelectronics (MIPRO), $201437^{\text {th }}$ International Convention on IEEE, 13041308.

Ekinci, M. 2006. Human identification using gait. Turkish Journal of Electrical Engineering \& Computer Sciences 14(2): 267-291.

Ekinci, M., Aykut, M. \& Gedikli, E. 2007, July. Gait recognition by applying multiple projections and kernel PCA. In International Workshop on Machine Learning and Data Mining in Pattern Recognition, Berlin, Heidelberg, 727-741.

Escalera, S., Pujol, O. \& Radeva, P. 2010. Error-correcting output codes library. Journal of Machine Learning Research 11(Feb): 661-664.

Escalera, S. \& Pujol, O. 2006. ECOC-ONE: A novel coding and decoding strategy. In null, 578-581.

Fazli, S., Askarifar, H. \& Tavassoli, M.J. 2011. Gait recognition using svm and lda. In Proc. of Int. Conf. on Advances in Computing, Control, and Telecommunication Technologies, 1-4.

Gianaria, E., Balossino, N., Grangetto, M. \& Lucenteforte, M. 2013. Gait characterization using dynamic skeleton acquisition. In Multimedia Signal Processing (MMSP) 2013, 440-445.

Gianaria, E., Marco, G., Maurizio, L. \& Nello, B. 2014. Biometric Authentication. Lecture Notes in Computer Science, 16-27.

Hall, M. 2015. Online Recognition of Malayalam Handwritten, $1-5$.
Han, J. \& Bir, B. 2006. Individual recognition using gait energy image. IEEE Transactions on Pattern Analysis and Machine Intelligence 28: 316-322.

Han, J. \& Bhanu, B. 2004. Statistical Feature Fusion for GaitBased Human Recognition. Proceedings of the 2004 IEEE Computer Society Conference on Computer Vision and Pattern Recognition, 2004. CVPR 2004. 2: 0-5.

Hofmann, M., Geiger, J., Bachmann, S., Schuller, B. \& Rigoll, G. 2014. The tum gait from audio, image and depth (gaid) database: Multimodal recognition of subjects and traits. Journal of Visual Communication and Image Representation 25(1): 195-206.

Hofmann, M. \& Gerhard, R. 2013. Exploiting Gradient Histograms for Gait-Based Person Identification. 16th IEEE International Conference on Image Processing (ICIP), 4171-4175.

Hosseini, N.K. \& Nordin, M.J. 2013. Human gait recognition: A silhouette based approach. Journal of Automation and Control Engineering 1(2): 259-267.

Hossin, M. \& Sulaiman, M.N. 2015. A Review on evaluation metrics for data classification evaluations. International Journal of Data Mining \& Knowledge Management Process 5(2): 1-11.

Jiang, S., Wang, Y., Zhang, Y. \& Sun, J., 2014. Real time gait recognition system based on kinect skeleton feature. Asian Conference on Computer Vision, 46-57.

Kochhar, A., Gupta, D., Hanmandlu, M. \& Vasikarla, S. 2013. Silhouette based gait recognition based on the area features using both model free and model based approaches. Technologies for Homeland Security (HST), 547-551.

Kouropteva, O., Okun, O. \& Pietikäinen, M. 2003. Supervised locally linear embedding algorithm for pattern recognition. In Iberian Conference on Pattern Recognition and Image Analysis, 386-394.

Kusy, M. 2004. Application of SVM to Ovarian Cancer. Artificial Intelligence and Soft Computing, 1020-1025.

Lam, T.H., Cheung, K.H. \& Liu, J.N. 2011. Gait flow image: A silhouette-based gait representation for human identification. Pattern Recognition 44(4): 973-987.

Machado-Molina, M., Bonninger, I., Dutta, M.K., Kutzner, T. \& Travieso, C.M. 2014. Gait-based recognition of humans using kinect camera. Recent Advances in Computer Engineering, Communications and Information Technology, Espanha, 63-71.

Meddeb, M. \& Hichem, K. 2015. Speech emotion recognition based on Arabic features, in 15th ISDA 46-51.

Naresh Kumar, M. S. \& Venkatesh Babu, R. 2012.Human gait recognition using depth camera: A Covariance Based Approach. Proceedings of the Eighth Indian Conference on Computer Vision, Graphics and Image Processing, 1-6.

Prathap, C. \& Sakkara, S. 2015. Gait recognition using skeleton data. 2015 International Conference on Advances in Computing, Communications and Informatics, ICACCI 2015, 2302-2306. 
Preis, J., Moritz, K., Martin, W. \& Claudia, L.P. 2012. Gait recognition with kinect. Workshop on Kinect in Pervasive Computing at Pervasive 2012.

Purnama, B., Wisesti, U.N., Nhita, F., Gayatri, A. \& Mutiah, T., 2015. A classification of polycystic Ovary Syndrome based on follicle detection of ultrasound images. Information and Communication Technology (ICoICT), 396-401.

Rida, I., Jiang, X. \& Marcialis, G.L., 2016. Human body part selection by group lasso of motion for model-free gait recognition. IEEE Signal Processing Letters 23(1): 154-158.

Sadhu, A.K., Saha, S., Konar, A. \& Janarthanan, R., 2014. Person identification using Kinect sensor. In Control, Instrumentation, Energy and Communication (CIEC), 214-218.

Sahak, R., Mansor, W., Lee, Y.K., Zabidi, A. \& Yassin, A.I.M. 2011. Orthogonal least square and optimized support vector machine with polynomial kernel for classifying asphyxiated infant cry. Signal and Image Processing Applications (ICSIPA), 104-108.

Sahay, T., Aggarwal, A., Bansal, A. \& Chandra, M., 2015. SVM and ANN: A comparative evaluation. Next Generation Computing Technologies (NGCT), 960964.

Sengupta, S., Halder, U., Panda, R. \& Chowdhury, A.S. 2013. A frequency domain approach to silhouette based gait recognition. Computer Vision, Pattern Recognition, Image Processing and Graphics (NCVPRIPG), 1-4.

Sharif, Z.A.M., Othman, M. \& Theong, T.S. 1991. Isolated digit recognition in Malaysian Language. Jurnal Kejuruteraan, 3: 41-46.

Shirke, S., Pawar, S.S. \& Shah, K. 2014. Literature review: Model free human gait recognition. Communication Systems and Network Technologies (CSNT), 891-895.

Sinha, A., Chakravarty, K. \& Bhowmick, B. 2013. Person identification using skeleton information from Kinect. ACHI 2013, The Sixth International Conference on Advances in Computer-Human Interactions, 101-108.

Sinha, A., Das, D., Chakravarty, K., Konar, A. \& Dutta, S. 2014. Kinect based people identification system using fusion of clustering and classification." International Conference on Computer Vision Theory and Applications (VISAPP) 3: 171-79.
Sivapalan, S., Chen, D., Denman, S., Sridharan, S. \& Fookes, C. 2011. Gait energy volumes and frontal gait recognition using depth images. Biometrics (IJCB), 2011 International Joint Conference on IEEE, 1-6.

Sivapalan, S., Chen, D., Denman, S., Sridharan, S. \& Fookes, C. 2012. The backfilled gei-a cross-capture modality gait feature for frontal and side-view gait recognition. Digital Image Computing Techniques and Applications (DICTA), 2012 International Conference on IEEE, 1-8.

Sokolova, M. \& Guy, L. 2009. A systematic analysis of performance measures for classification tasks. Information Processing and Management 45(4): $427-$ 437.

Spline Curves. Available at https://people.cs.clemson. edu/ dhouse/courses/405/notes/splines.pdf [Accessed 01-May-2016].

Yan, X. \& Wang, Y., 2009. A feather and down category recognition System Based on GA and SVM. Education Technology and Computer, 2009. ICETC'09. International Conference on IEEE, 128-132.

Yoo, J.H. \& Nixon, M.S. 2011. Automated markerless analysis of human gait motion for recognition and classification. EtTRI Journal 33(2): 259-266.

Rohilah Sahak

* Nooritawati Md Tahir

Ahmad Ihsan Mohd Yassin

Fadhlan Hafizhelmi Kamaruzaman

Faculty of Electrical Engineering

Universiti Teknologi MARA (UiTM)

Shah Alam, Selangor, Malaysia.

*Corresponding author; email: nooritawati@ieee.org

Received date: $21^{\text {st }}$ January 2018

Accepted date: $15^{\text {th }}$ May 2018

Online first date: $1^{\text {st }}$ September 2018

Published date: $31^{\text {st }}$ October 2018 